\title{
FTY720 Protects Against Ischemia-Reperfusion Injury by Preventing the Redistribution of Tight Junction Proteins and Decreases Inflammation in the Subacute Phase in an Experimental Stroke Model
}

\author{
Zifeng Wang ${ }^{1} \cdot$ Kei Higashikawa ${ }^{2} \cdot$ Hironobu Yasui ${ }^{2} \cdot$ Yuji Kuge $^{2} \cdot$ Yusuke Ohno $^{3} \cdot$ Akio Kihara $^{3} \cdot$ Yenari A. Midori ${ }^{4}$. \\ Kiyohiro Houkin ${ }^{1} \cdot$ Masahito Kawabori ${ }^{1}$ (i)
}

Received: 1 May 2019 / Revised: 22 January 2020 / Accepted: 12 February 2020 / Published online: 27 February 2020

(C) The Author(s) 2020

\begin{abstract}
Injury due to brain ischemia followed by reperfusion (I/R) may be an important therapeutic target in the era of thrombectomy. FTY720, a widely known sphingosine-1-phosphate receptor agonist, exerts various neuroprotective effects. The aim of this study was to examine the protective effect of FTY720 with respect to I/R injury, especially focusing on blood-brain barrier (BBB) protection and anti-inflammatory effects. Male rats were subjected to transient ischemia and administered vehicle or 0.5 or $1.5 \mathrm{mg} / \mathrm{kg}$ of FTY720 immediately before reperfusion. Positron emission tomography (PET) with $\left[{ }^{18} \mathrm{~F}\right] \mathrm{DPA}-714$ was performed 2 and 9 days after the insult to serially monitor neuroinflammation. Bovine and rat brain microvascular endothelial cells (MVECs) were also subjected to oxygen-glucose deprivation (OGD) and reperfusion, and administered FTY720, phosphorylated-FTY720 (FTY720-P), or their inhibitor. FTY720 dose-dependently reduced cell death, the infarct size, cell death including apoptosis, and inflammation. It also ameliorated BBB disruption and neurological deficits compared to in the vehicle group. PET indicated that FTY720 significantly inhibited the worsening of inflammation in later stages. FTY720-P significantly prevented the intracellular redistribution of tight junction proteins but did not increase their mRNA expression. These results suggest that FTY720 can ameliorate I/R injury by protecting the BBB and regulating neuroinflammation.
\end{abstract}

Keywords FTY720 · Sphingosine-1-phosphate receptor · Brain ischemia-reperfusion · Blood-brain barrier · Inflammation . Apoptosis

Electronic supplementary material The online version of this article (https://doi.org/10.1007/s12975-020-00789-x) contains supplementary material, which is available to authorized users.

\section{Masahito Kawabori}

masahitokawabori@yahoo.co.jp

1 Department of Neurosurgery, Graduate School of Medicine, Hokkaido University, Kita 15, Nishi 7, Kita-ku,

Sapporo, Hokkaido 060-8638, Japan

2 Central Institutes of Isotope Science (Laboratory of Integrated Molecular Imaging, Department of Biomedical Imaging, Graduate School of Biomedical Science and Engineering), Hokkaido University, Sapporo, Hokkaido, Japan

3 Laboratory of Biochemistry, Faculty of Pharmaceutical Sciences, Hokkaido University, Sapporo, Hokkaido, Japan

4 Department of Neurology, University of California, San Francisco and the San Francisco Veterans Affairs Medical Center, San Francisco, CA, USA

\section{Introduction}

Ischemic stroke is a major health problem worldwide and is associated with extremely high morbidity and mortality [1]. Recent advances in thrombectomy have brought forth a new era for the treatment of acute ischemic stroke [2-4]. Thrombectomy can achieve higher rates of angiographic revascularization and provides better functional outcomes even up to $24 \mathrm{~h}$ after symptom onset. Although recanalization by thrombectomy can restore cerebral blood flow, many patients still do not show sufficient clinical recovery [2-4]. Ischemiareperfusion ( $\mathrm{I} / \mathrm{R})$ injury is thought to be one of the major reasons for unfavorable outcomes following thrombolysis and thrombectomy. I/R injury is defined as a biochemical cascade that exacerbates damage in the re-perfused brain tissue [5]. It can affect pathways leading to excitotoxicity, apoptosis, inflammation, free radical production, and loss of blood-brain barrier (BBB) integrity. These insults subsequently induce immune cell infiltration and extracellular 
matrix damage, resulting in brain edema and hemorrhagic transformation [6]. Thus, studies are urgently needed to understand these mechanisms and treatments to maximize the therapeutic effect of thrombectomy. FTY720, a novel immunomodulator that acts as a sphingosine-1-phosphate (S1P) receptor agonist, was recently approved for the treatment for multiple sclerosis by the US Food and Drug Administration. In vivo, FTY720 can be rapidly phosphorylated, generating its biologically active form, namely phosphorylated-FTY720 (FTY720-P), which subsequently binds four of the five S1P receptors to regulate multiple cellular events [7]. The effect of FTY720 includes the induction of systemic lymphopenia, inhibition of inflammatory responses, and downregulation of intravascular adhesion molecules. Recently, several studies have shown that FTY720 protects organs from I/R injury, such as the liver [8,9], kidney [10, 11], heart [12-14], and brain [15-19]. However, the mechanisms underlying the BBBprotective effects of FTY720 and time course of inflammatory modulation have not been fully elucidated. The aim of this study was to examine the protective effect of FTY 720 on brain $\mathrm{I} / \mathrm{R}$, with a focus on $\mathrm{BBB}$ preservation and the regulation of inflammatory reactions.

\section{Materials and Methods}

All experimental protocols were approved by the Animal Studies Ethical Committee at Hokkaido University Graduate School of Medicine (reference number 17-0066). All procedures used in the present study were performed in accordance with the institutional guidelines for animal experimentation and the Guidelines for Proper Conduct of Animal Experiments by the Science Council of Japan and the ARRIVE (Animal Research: Reporting In Vivo Experiments) guidelines.

\section{Experimental Animals}

In total, 123 wild-type 8-week-old male Sprague-Dawley rats (CLEA Japan, Inc., Tokyo, Japan) weighing 260-300 g were used for the experiments. Male rats were employed to eliminate any estrogen-mediated neuroprotective effects on ischemic injury. Animals were housed in a controlled environment $\left(25{ }^{\circ} \mathrm{C}, 50 \%\right.$ humidity, and a 12 -h light-dark cycle) and allowed free access to food and water. All experimental animals were randomly divided into the following groups: middle cerebral artery occlusion (MCAO) with vehicle treatment $(n=60)$, MCAO with low-dose $(0.5 \mathrm{mg} / \mathrm{kg})$ FTY720 treatment $(n=29)$, and MCAO with high-dose of FTY720 $(1.5 \mathrm{mg} / \mathrm{kg})$ treatment $(n=28)$.

\section{tMCAO Model}

Transient focal cerebral ischemia was induced for $2 \mathrm{~h}$ using a silicone rubber-coated nylon filament inserted into the right middle cerebral artery (MCA) as previously described [20-22]. Briefly, rats were anesthetized with isoflurane at initial and maintenance concentrations of $4.0 \%$ and $2.0 \%$, respectively, in $70 \% \mathrm{~N}_{2} \mathrm{O}$ and $30 \% \mathrm{O}_{2}$ gas, thorough a facial mask. The rectal temperature of experimental animals was maintained between 36.5 and $37.5^{\circ} \mathrm{C}$ throughout the procedures using an automated heat pad. Transient focal ischemia was induced by occluding the right MCA using a silicone rubber-coated nylon filament with a tip diameter of $0.37 \mathrm{~mm}$ (Doccol Corp., Redlands, CA, USA). The right carotid artery (CA) was surgically exposed, and the external CA was then ligated, cut, and reversed proximally. The filament was inserted into the external CA and advanced into the internal CA to block the origin of the MCA. Cerebral blood flow (CBF) measurements and neurological scoring assessments were performed to verify successful MCAO. CBF in the MCA region $(5 \mathrm{~mm}$ lateral to the middle line and $2 \mathrm{~mm}$ posterior to the bregma) was measured before and after MCAO by laser Doppler flowmetry (OMEGA FLOW FLO-C1; OMEGAWAVE, Tokyo, Japan). Rats with a relative reduction of CBF higher than $30 \%$ compared to preoperative data and/or a modified Bederson score of 2 points or lower were excluded from the experiment. After the 2-h transient middle cerebral artery occlusion (tMCAO), the filament was gently withdrawn to provide reperfusion.

\section{Administration of FTY720}

The low-dose group was administered $0.5 \mathrm{mg} / \mathrm{kg}$ FTY720 (Cayman Chemical, Ann Arbor, MI, USA) diluted in saline, whereas the high-dose group was treated with $1.5 \mathrm{mg} / \mathrm{kg}$ FTY720. FTY720 was intraperitoneally injected immediately before reperfusion.

\section{Neurological Score}

An 18-point modified neurological severity score (mNSS) and four-point modified Bederson score were used to evaluate neurological deficits $[23,24]$. Neurological evaluation was performed before and after MCA occlusion on days 1, 3, 5, and 7.

\section{Evaluation of Brain Infarct Volume}

The infarct volume was evaluated 7 days after reperfusion by 2,3,5-triphenyltetrazolium chloride (TTC) staining. Rats were deeply anesthetized to prevent pain and discomfort at the time of sacrifice. They were then sacrificed by spinal dislocation and decapitation 7 days after reperfusion. The brains were 
then removed and placed immediately on ice for $5 \mathrm{~min}$. Brain samples were sliced into six uniform coronal sections of $2-\mathrm{mm}$ thickness, and sections were stained with $2 \%$ TTC for $15 \mathrm{~min}$ $[25,26]$. The infarct lesions were photographed and quantitatively analyzed with ImageJ software (ImageJ $1.37 \mathrm{v}$; NIH, Bethesda, MD, USA) by an observer blinded to the groups. Unstained areas (pale color) were defined as ischemic lesions, and the infarct size was calculated based on the following formula to avoid the effect of brain edema [27].

Brain infarct $(\%)=($ contralateral hemispheric volume - ip silateral non-infarct volume)/contralateral hemispheric volume.

\section{Immunohistochemistry and Apoptosis Assay}

Paraffin sections of brain tissue fixed in 4\% paraformaldehyde were used for immunohistochemistry. Four-micrometer-thick coronal sections at the level of the striatum were prepared. The antibodies used for immunohistochemistry of neuroinflammation were anti-Ibal (1:1500 at room temperature for $1 \mathrm{~h}$; 01919741, Wako, Japan) and anti-CD68 (1:1000 at room temperature for $1 \mathrm{~h}$; MCA341GA, BIO-RAD). After incubation with the primary antibody, the sections were treated with Histofine ${ }^{\circledR}$ Simple StainTM Rat MAX PO (Nichirei Biosciences, Tokyo, Japan) for $1 \mathrm{~h}$. Next, the DAB chromogen of the DAB Substitute Kit (Nichirei Biosciences) was applied for 10-30 s to obtain the chromogenic signal in accordance with the manufacturer's instructions. For immunofluorescence staining of apoptotic cells, TUNEL was performed using the ApopTag Fluorescein In Situ Apoptosis Detection Kit (S7110, Chemicon International, Temecula, CA, USA) according to the manufacturer's protocol. Ten regions of interest (ROIs) $(100 \times 100 \mu \mathrm{m})$ were randomly placed on the area adjacent to the boundary zone of the infarct (peri-infarct) to count the positive cells [28].

\section{PET Scans and Autoradiography Imaging}

A neural inflammatory biomarker translocator protein (TSPO) was serially monitored on days 2 and 9 by positron emission tomography (PET) using the TSPO ligand $\left[{ }^{18} \mathrm{~F}\right] \mathrm{DPA}-714$ as previously reported [29]. PET imaging was serially performed with the same rats ( $n=4$ per group); images were obtained using the Inveon small animal imaging system (Siemens Medical Solutions, Knoxville, TN, USA). The PET component consisted of $1.5 \times 1.5 \times 10 \mathrm{~mm}$ lutetium oxyorthosilicate crystal elements with a ring diameter of $16.1 \mathrm{~cm}$, providing a $10-\mathrm{cm}$ transaxial and $12.7-\mathrm{cm}$ axial field of view [30]. Rats anesthetized with isoflurane were injected with $13.2 \pm$ $0.8 \mathrm{MBq}[18 \mathrm{~F}] \mathrm{DPA}-714$ via the tail vein. They were then returned to their cages and allowed to move freely for $30 \mathrm{~min}$. All performance measurements were set to a coincidence window of $3.432 \mathrm{~ns}$ and energy window of 350
$650 \mathrm{keV}$. Delayed events were subtracted from prompt events to correct for random events. The image matrix was $256 \times$ $256 \times 159$, resulting in a voxel size of $0.385 \times 0.385 \times$ $0.796 \mathrm{~mm}(512 \times 512 \times 159$ matrix, $0.215 \times 0.215 \times$ $0.796 \mathrm{~mm}$ for spatial resolution measurements) [31]. ROI analysis was conducted in the area of infarction, and the control was symmetrically placed in the contralateral hemisphere. Next, counting rates were converted to standardized uptake values (SUVs) and the ratio of ipsilateral to contralateral radioactivity was calculated using IDL (Research Systems, CO, USA) and ASIPro VM (Concorde Microsystems, Knoxville, TN, USA). For autoradiography experiments, rats were sacrificed at $90 \mathrm{~min}$ after [18F]DPA-714 injection. The brains were quickly removed and cut into six 2-mm-thick coronal slices. The second and fourth slices were exposed to a phosphor imaging plate (Fuji Photo Film Co., Ltd., Tokyo, Japan) together with a set of calibrated standards. After exposure, the imaging plate was scanned with an FLA 7000 BioImaging Analyzer (Fujifilm Life Science) and images were analyzed using Multi Gauge V3.2 (Fujifilm Life Science).

\section{Evaluation of In Vivo BBB Integrity}

Evans blue dye and fluorescent dextran were used to measure BBB disruption [21,32]. When intravenously injected, Evans blue binds to albumin to form a high molecular weight serum protein complex ( $68 \mathrm{kDa}$ ), and two different types of fluorescent dextran ( 3 and $10 \mathrm{kDa})$ were also applied to determine the size selectivity of BBB leakage. Immediately after reperfusion, $4 \mathrm{~mL} / \mathrm{kg}$ of $2 \%$ Evans blue (Wako, Osaka, Japan) or $50 \mathrm{mg} / \mathrm{kg}$ fluorescent dextran (FD4; $3 \mathrm{kD}, \mathrm{FD} 10 \mathrm{~S} ; 10 \mathrm{kD}$, Sigma, St. Louis, MO, USA) diluted in saline was intravenously administered. The amount of Evans blue leakage was assessed by brain supernatant absorbance, and fluorescent dextran was assessed by immunohistochemistry analysis as previously reported [21, 32]. Briefly, at $24 \mathrm{~h}$ after reperfusion, the rats were sacrificed and perfused with saline through the left ventricle to wash out the intravascular Evans blue or fluorescent dextran. The right cerebral hemisphere was then removed and homogenized in $3 \mathrm{~mL}$ of $50 \%$ trichloroacetic acid solution and then centrifuged (12,000 rpm for $10 \mathrm{~min})$. The absorbance of the supernatant at $620 \mathrm{~nm}$ was measured using a spectrophotometer (TECAN Japan, Kanagawa, Japan). The tissue contents of Evans blue were quantified from a linear standard curve and expressed as micrograms per gram of brain tissue. Fluorescent dextran leakage was measured by immunohistochemistry. Four-micrometer-thick paraffin coronal sections at the level of striatum were prepared. The sections were treated with a collagen IV antibody $\left(1: 200,4{ }^{\circ} \mathrm{C}\right.$ overnight, Proteintech, Rosemont, IL, USA) followed by Alexa Fluor 594 Goat Anti-Rabbit IgG Antibody (1:200, A11012, Invitrogen). Extra-vascular dextran leakage was assessed in randomly selected 5 non-overlapping fields $(700 \times 560 \mu \mathrm{m})$ 
at around the peri-infarct area. The amount of leakage was semi-quantitatively analyzed by the following formula: vascular leakage $=$ area of leakage (fluorescent dextran)/microvascular area (collagen IV).

\section{Cell Culture}

Bovine brain microvascular endothelial cells (BBMVECs) were purchased from Cell Applications, Inc. (San Diego, CA, USA), and the rat microvascular BBB kit was purchased from PharmaCo-Cell, Inc. (Nagasaki, Japan) for in vitro experiments. Next, 100-mm cell culture dishes (Thermo Fisher Scientific, Waltham, MA, USA), 2-well chamber slides (Watson Biolab, Kobe, Japan), and a permeability kit (PharmaCo-Cell) were used to culture cells in high-glucose Dulbecco's modified Eagle's medium (DMEM; Nacalai Tesque, Inc., Kyoto, Japan), containing $10 \%$ fetal bovine serum (FBS) and $100 \mathrm{U} / \mathrm{mL}$ penicillin G. The cells were maintained in an incubator at $37{ }^{\circ} \mathrm{C}$ with $5 \% \mathrm{CO}_{2}$, and subconfluent monolayers of early passage cells (P2-4), or cells condition instructed by manufacture were used for each experiment [29].

\section{OGD and Reperfusion Model}

The cells were exposed to oxygen-glucose deprivation (OGD) for $4 \mathrm{~h}$ and then returned to normoxic conditions $\left(37^{\circ} \mathrm{C}, 5 \%\right.$ $\mathrm{CO}_{2}$ ) in high-glucose medium without FBS for $4 \mathrm{~h}$, as previously described [20]. Before OGD, the cells were cultured in high-glucose DMEM with activated charcoal-filtered FBS for $12 \mathrm{~h}$, followed by high-glucose DMEM without FBS for another $12 \mathrm{~h}$ to eliminate S1P contained in the FBS. OGD was achieved under hypoxic conditions $\left(1 \% \mathrm{O}_{2}, 5 \% \mathrm{CO}_{2}\right.$, and $94 \% \mathrm{~N}_{2}$ at $37{ }^{\circ} \mathrm{C}$ ) generated by a hypoxia workstation (InvivoO2 300, Baker Ruskinn, Sanford, ME, USA) and DMEM without glucose. This medium was prepared to be hypoxic by overnight incubation under hypoxic conditions. The cells were then randomly divided into the following groups ( $n=5$ per group): (1) control group, no OGD; (2) vehicle group, OGD without treatment; (3) FTY720 group, OGD with $100 \mathrm{nM}$ of FTY720 added to the culture medium immediately before reperfusion; (4) FTY720-P group, OGD with $100 \mathrm{nM}$ of FTY720-P (Cayman Chemical) added to the culture medium immediately before reperfusion; (5) FTY720$\mathrm{P}$ and pertussis toxin (PTX) group, OGD with FTY720-P $(100 \mathrm{nM})$ and PTX $(100 \mathrm{ng} / \mathrm{mL}$, Fujifilm) added to the culture medium immediately before reperfusion. As FTY720 must be converted to FTY720-P to be physiologically active and BBMVECs are thought to not possess sufficient enzymes to convert FTY720 to FTY720-P, FTY720-P was also applied to the cells. PTX is an inhibitor of the Gi-coupled S1P receptor and acts as an antagonist of FTY720 and FTY720-P.

\section{Evaluation of In Vitro BBB Integrity}

A rat microvascular BBB kit (PharmacoCell, Nagasaki, Japan) was used to measure in vitro BBB integrity according to the manufacturer's instructions. Briefly, the cells were cultured as previously mentioned and transendothelial electrical resistance (TEER) was measured just before starting OGD to ensure that the resistance was higher than $150 \Omega$, which indicates a sufficient barrier function (data not shown). OGD and reperfusion were performed as previously mentioned, and Evans blue preconjugated with serum albumin or FITC dextran $(3$ and $5 \mathrm{kD})$ were added to the upper wells $(0.2 \mathrm{mg} /$ $200 \mu \mathrm{L}$ ). The medium in the lower well was collected 30 min later. The concentration of Evans blue in the lower well was measured with a spectrophotometer (TECAN, Männedorf, Switzerland) as previously described, and the FITC concentration was measured with a fluorescence plate reader (Spectra Max paradigm, Molecular Devices, Sunnyvale, CA, USA). The permeant diffusivity of Evans blue or FITC dextran was measured, and the relative ratio of diffusion was qualitatively analyzed between groups as previously reported [33].

\section{Real-Time PCR and Western Blotting Analysis}

The expression of tight and adheres junctional proteins including ZO-1, occludin, claudin-5, and VE-cadherin after OGD treatment was examined by real-time PCR. RNA was extracted from the cells using an AllPrep RNA Mini Kit (Qiagen, Hilden, Germany). First-strand cDNA was synthesized using a PrimeScript ${ }^{\circledR}$ II 1st strand cDNA Synthesis Kit (Takara, Shiga, Japan) in accordance with the manufacturers' recommendations. The relative differences in gene expression between groups were expressed based on cycle time $(\mathrm{Ct})$ values. The $\mathrm{Ct}$ values of the genes of interest were first normalized to $G A P D H$ in the same sample and then relative differences between control and treatment groups were obtained. The primer sequences for real-time PCR are shown in Supplementary Table 1.

Western blotting was performed using an anti-ERK1/2 monoclonal antibody (1:2000, Thermo Fisher Scientific) to evaluate downstream signaling from the $\mathrm{S}_{1} \mathrm{P}_{1}$ receptor. Proteins were extracted from the BBMECs in 100-mm culture dishes ( $n=6$ per group; control, vehicle, FTY720, FTY720-P, FTY720-P, and PTX). The cells were collected and homogenized in RIPA lysis buffer (Santa Cruz Biotechnology, Dallas, TX, USA) and an equal amount of total protein $(10 \mu \mathrm{g})$ was electrophoresed on a NuPage 4-12\% Bis-Tris Gel (Life Technologies, Carlsbad, CA, USA). Proteins were then blotted onto a cellulose membrane using the iBlot2 (Thermo Fisher Scientific) following the manufacturer's protocol. The membrane was blocked with ECL Prime blocking agent (GE Healthcare Life Science, Little Chalfont, UK) in PBS 
containing $0.05 \%$ Tween-20 at room temperature for $1 \mathrm{~h}$, followed by incubation with primary antibody at $4{ }^{\circ} \mathrm{C}$ overnight. After washing with PBS containing $0.05 \%$ Tween-20, the membrane was incubated with a peroxidase-conjugated secondary antibody at room temperature for $1 \mathrm{~h}$. Labeled proteins were detected by chemical luminescence (ECL Advanced Western Blotting Detection Kit; GE Healthcare Life Science). Immunoblots were quantified by densitometry using software provided by the imaging system (ChemiDoc; Bio-Rad, Hercules, CA, USA).

\section{Immunocytofluorescence of BBB Components}

Redistribution of the junctional proteins (ZO-1, VE-cadherin, occludin, claudin-5) was examined by immunocytofluorescence. BBMEVCs or rat brain microvascular endothelial cells were treated with OGD followed by drug administration as previously mentioned. The cells were then fixed with $4 \%$ paraformaldehyde for $15 \mathrm{~min}$, permeabilized with $0.1 \%$ Triton X-100 for $10 \mathrm{~min}$, and blocked with $3 \%$ bovine serum albumin for $30 \mathrm{~min}$. The cells were then incubated with Blockace (DS Pharma Biomedical, Inc., Osaka, Japan) for $1 \mathrm{~h}$ to block nonspecific reactions. The cells were treated with anti-VE-cadherin (1:100, catalog number: PA5-17401, Thermo Fisher Scientific), anti-ZO-1 (1:100, catalog number: 33-9100, Thermo Fisher Scientific), anti-occludin (1:100, MBS9608114, MyBioSource), and anti-claudin-5 (1:100, GTX49371, GenTex) as primary antibodies at room temperature for $1 \mathrm{~h}$, followed by incubation with Alexa Fluor 488 Goat Anti-Rabbit IgG Antibody (1:200, Life Technologies) as the secondary antibody at room temperature for $1 \mathrm{~h}$ and DAPI staining. Stained images were visualized and photographed with a fluorescence microscope (BZ X-700, Keyence, Osaka, Japan). Ten nonoverlapping fields $(700 \times 560 \mu \mathrm{m})$ were randomly selected, and numbers and ratio of the cells with junctional protein at the cell border or in the cytoplasm were semi-quantitively analyzed [34-36].

\section{Statistical Analysis}

All data were collected and analyzed by investigators blinded to all randomized animal and cell experimental groups. The data are presented as the mean \pm standard deviation. All statistical analyses were performed using JMP Pro 13 (Systat Software, San Jose, CA, USA). Continuous and noncontinuous data were compared using an unpaired $t$ test or Wilcoxon test between 2 groups and one-way analysis of variance followed by the Dunnett post hoc or Dunn test for multiple comparisons. Sample sizes were selected based on preliminary experiments. Briefly, in one-way analysis of variance study, sample sizes of 10,10 , and 20 were obtained from the 3 groups to compare the means. The total sample of $40 \mathrm{sub}-$ jects achieves $100 \%$ power for detecting differences among the means versus the alternative of equal means using an $F$ test with a 0.0500 significance level. The size of the variation in the means is represented by their standard deviation, which was 0.83 . The common standard deviation within a group was assumed to be 1.00. We used PASS 14.0.9 (PASS Software by NCSS, LLC) to compute the statistical power. A $p$ value < 0.05 was considered as statistically significant.

\section{Results}

\section{FTY720 Reduces Mortality and Infarct Size and Improves Functional Recovery}

There was a dose-dependent reduction in infarct size between groups, and a significant difference was detected between the high-dose FTY720 group and vehicle group $(P<0.01)$; specifically, FTY720 reduced the infarct size by approximately $55 \%$ in the high-dose group compared to in the vehicle group (Fig. 1a). Mortality was also reduced in a dose-dependent manner; specifically, the vehicle group showed a rate of $20.0 \%$, whereas the low-dose FTY720 group showed a rate of $8.0 \%$ and the high-dose group showed no mortality (Fig. 1b). A significant improvement in functional recovery was also observed in FTY720-treated animals, based on both the mNSS and modified Bederson Score, compared to in the vehicle group $(P<0.01)$. However, there was no difference in functional recovery between the low-dose and high-dose FTY720 groups (Fig. 1c, d).

\section{Activated Microglia/Macrophages and Apoptotic Cells Are Decreased in FTY720 Treatment Groups}

Activated microglia identified by Iba1 staining were counted at the infarct border (Fig. 2a). Further, the number of activated microglia (as identified by dark Ibal staining) was dosedependently decreased in the FTY720 treatment groups. Specifically, the number of activated microglia in the lowdose group was decreased by $50.0 \%(P<0.01)$ and that in the high-dose group was decreased by $64.4 \%(P<0.01) \mathrm{com}-$ pared to in the vehicle group. To investigate the effect of FTY720 on macrophage infiltration into damaged brains, CD68-positive cells were stained. Similar results were observed with Iba1-positive macrophages; specifically, FTY720 significantly decreased the number of CD68positive macrophages by $60.6 \%(P<0.01)$ in the low-dose group and by $84.2 \%(P<0.01)$ in the high-dose group compared to in the vehicle group (Fig. 2b). Furthermore, TUNEL staining revealed that apoptotic cells were significantly decreased by approximately $90 \%$ in both the low-dose $(91.4 \%$, $P<0.01)$ and high-dose $(90.5 \%, P<0.01)$ FTY720 groups compared to the number in the vehicle group (Fig. 2c). 
(a)
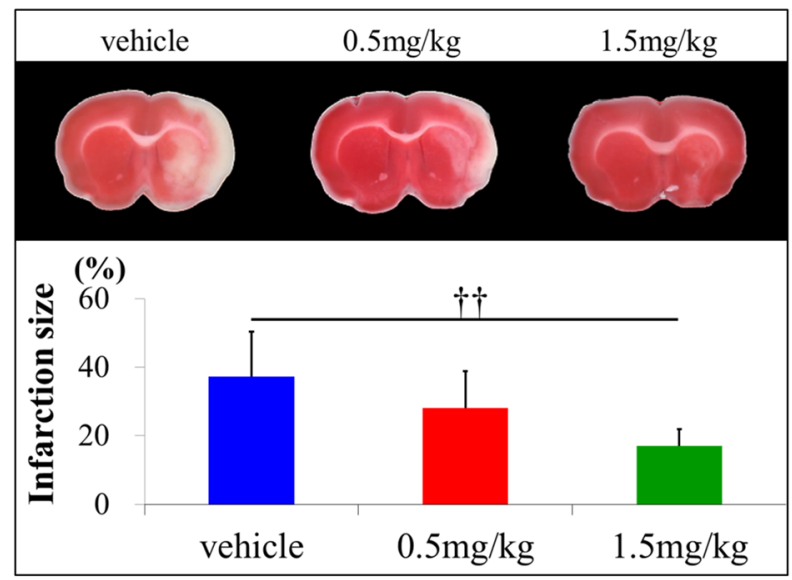

(c)

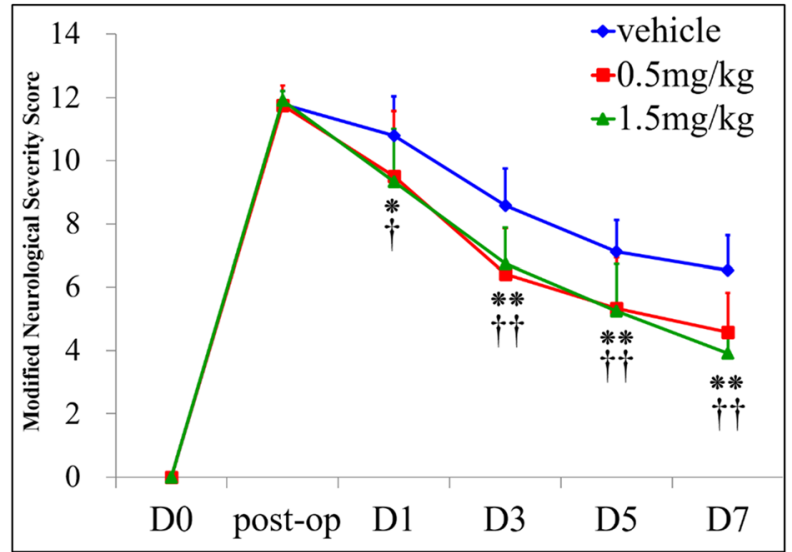

Fig. 1 Effect of FTY720 on infarct size, mortality ratio, and neurological recovery in a rat model of transient middle cerebral artery occlusion. a Animals (vehicle, $n=20 ; 0.5 \mathrm{mg} / \mathrm{kg}, n=6 ; 1.5 \mathrm{mg} / \mathrm{kg}, \mathrm{n}=6$ ) were sacrificed at day 7 after transient middle cerebral artery occlusion and 2,3,5-triphenyltetrazolium chloride staining of coronal brain sections was performed to analyze the efficacy of FTY 720 for reducing the infarct size. High-dose FTY720 $(1.5 \mathrm{mg} / \mathrm{kg})$ significantly reduced the infarct size compared to that in the vehicle group $(\dagger \dagger P<0.01$ versus $1.5 \mathrm{mg} / \mathrm{kg}$ ). b FTY720 showed a dose-dependent improvement in

\section{PET Imaging Revealed Suppression of Inflammatory Reactions by FTY720 at the Subacute Phase}

PET imaging was used to serially monitor the effect of FTY720 on inflammatory reactions (Fig. 3). $\left[{ }^{18}\right.$ F]DPA-714 PET showed no difference in inflammatory reactions among all groups 2 days after MCAO, as determined by the maximum (max) SUV and mean SUV (vehicle group: $\max$ SUV = $0.91 \pm 0.05$, mean SUV $=0.61 \pm 0.06$; low-dose FTY720 group: $\max \mathrm{SUV}=0.82 \pm 0.19$, mean $\mathrm{SUV}=0.49 \pm 0.16$; high dose FTY720 group: $\max \mathrm{SUV}=0.88 \pm 0.17$, mean SUV $=0.50 \pm 0.08$ ). However, 9 days after MCAO, whereas the SUV was significantly increased in the vehicle group, a dose-dependent reduction was observed in the FTY720 treatment groups (vehicle group: $\max \mathrm{SUV}=1.50 \pm 0.28$, mean SUV $=0.84 \pm 0.11$; low-dose FTY720 group: $\max$ SUV $=$ $1.25 \pm 0.13$, mean SUV $=0.65 \pm 0.08$; high-dose FTY720 (b)

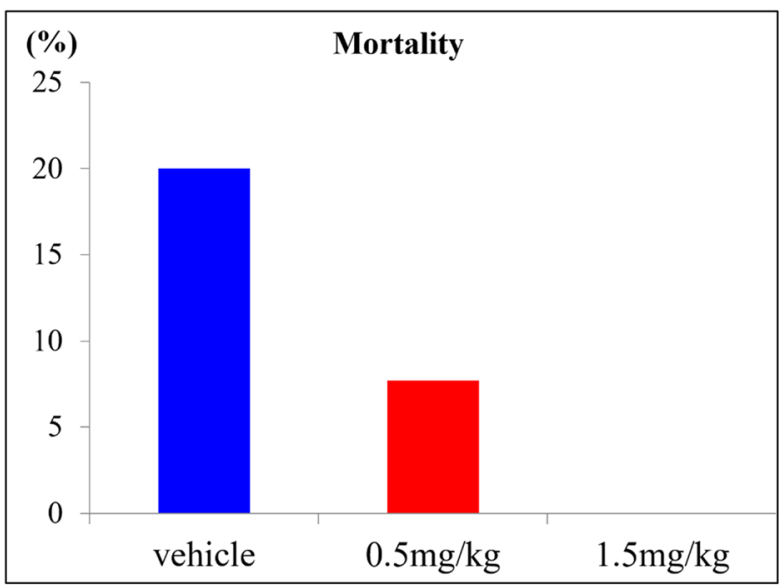

(d)

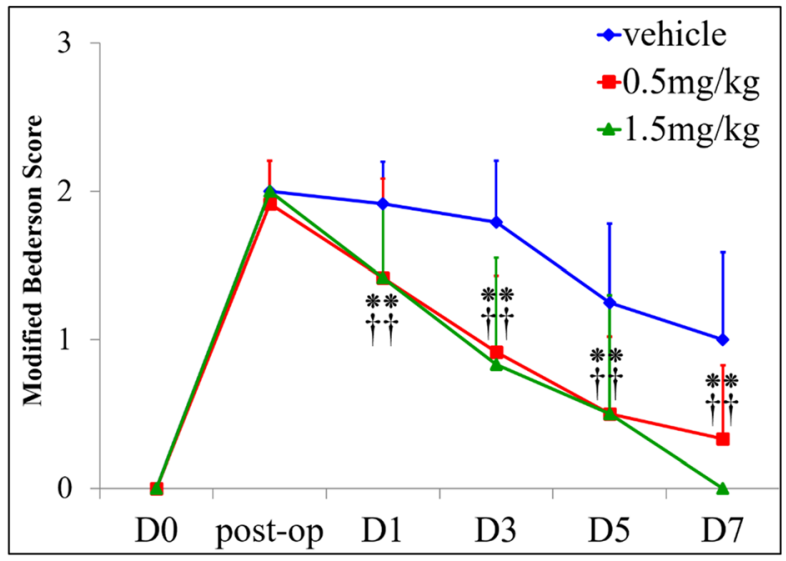

mortality ratio (vehicle, $n=36 ; 0.5 \mathrm{mg} / \mathrm{kg}, n=19 ; 1.5 \mathrm{mg} / \mathrm{kg}, n=12$ ). c, d Neurological function was assessed by the modified Neurological Severity Score (c) and modified Bederson score (d) in the vehicle, lowdose FTY720, and high-dose FTY720 groups (vehicle, $n=24 ; 0.5 \mathrm{mg} / \mathrm{kg}$, $n=12 ; 1.5 \mathrm{mg} / \mathrm{kg}, n=12$ ). Vehicle group, blue; $0.5 \mathrm{mg} / \mathrm{kg}$ group, red; $1.5 \mathrm{mg} / \mathrm{kg}$, green. Significant differences between the vehicle and treatment groups. Values are the mean; $* P<0.05$ versus $0.5 \mathrm{mg} / \mathrm{kg}, \dagger P<0.05$ versus $1.5 \mathrm{mg} / \mathrm{kg}, * * P<0.01$ versus $0.5 \mathrm{mg} / \mathrm{kg}, \dagger \dagger P<0.01$ versus $1.5 \mathrm{mg} / \mathrm{kg}$

group: $\max \mathrm{SUV}=0.98 \pm 0.37$, mean $\mathrm{SUV}=0.52 \pm 0.21$ ). Notably, no exacerbation in inflammation was observed in the high-dose FTY720 group during the experiment.

\section{FTY720 Ameliorates BBB Disruption In Vivo and In Vitro Model of Ischemia}

The effect of FTY720 against BBB disruption was assessed in both in vivo and in vitro model of ischemia. Evans blue extravasation was used to monitor in vivo rat brain BBB permeability for large molecule leakage. The content of dye in the ischemic hemisphere was significantly lower in both the highand low-dose FTY720 groups compared to in the vehicle group $(P<0.01$; Fig. 4a). Two different molecular weights of fluorescent dextran ( 3 and $10 \mathrm{kDa}$ ) were then used to assess BBB integrity for smaller molecules in the rat brain. The area ratio of fluorescent dextran extravascular leakage was dose- 
(a)
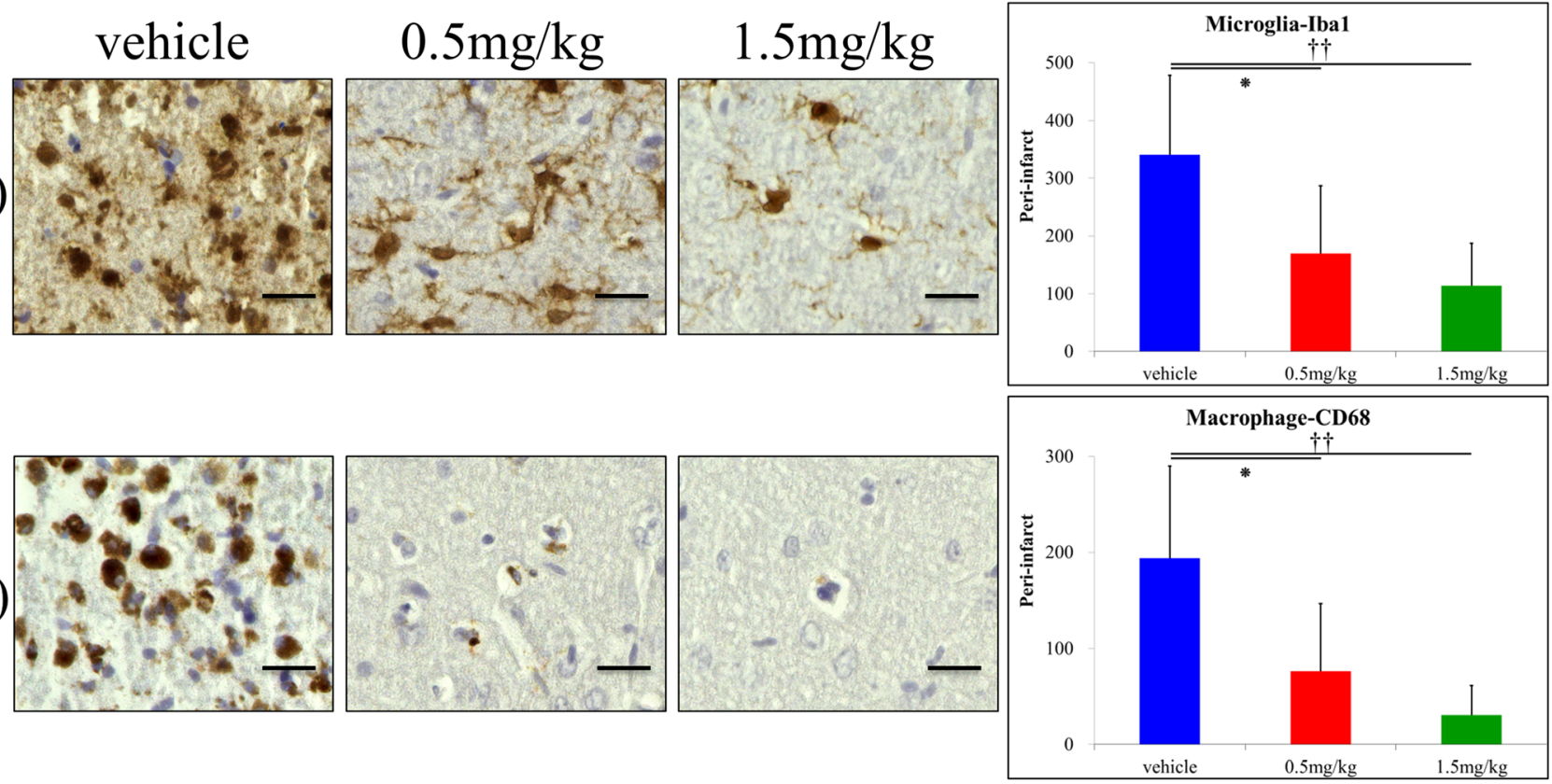

(b)
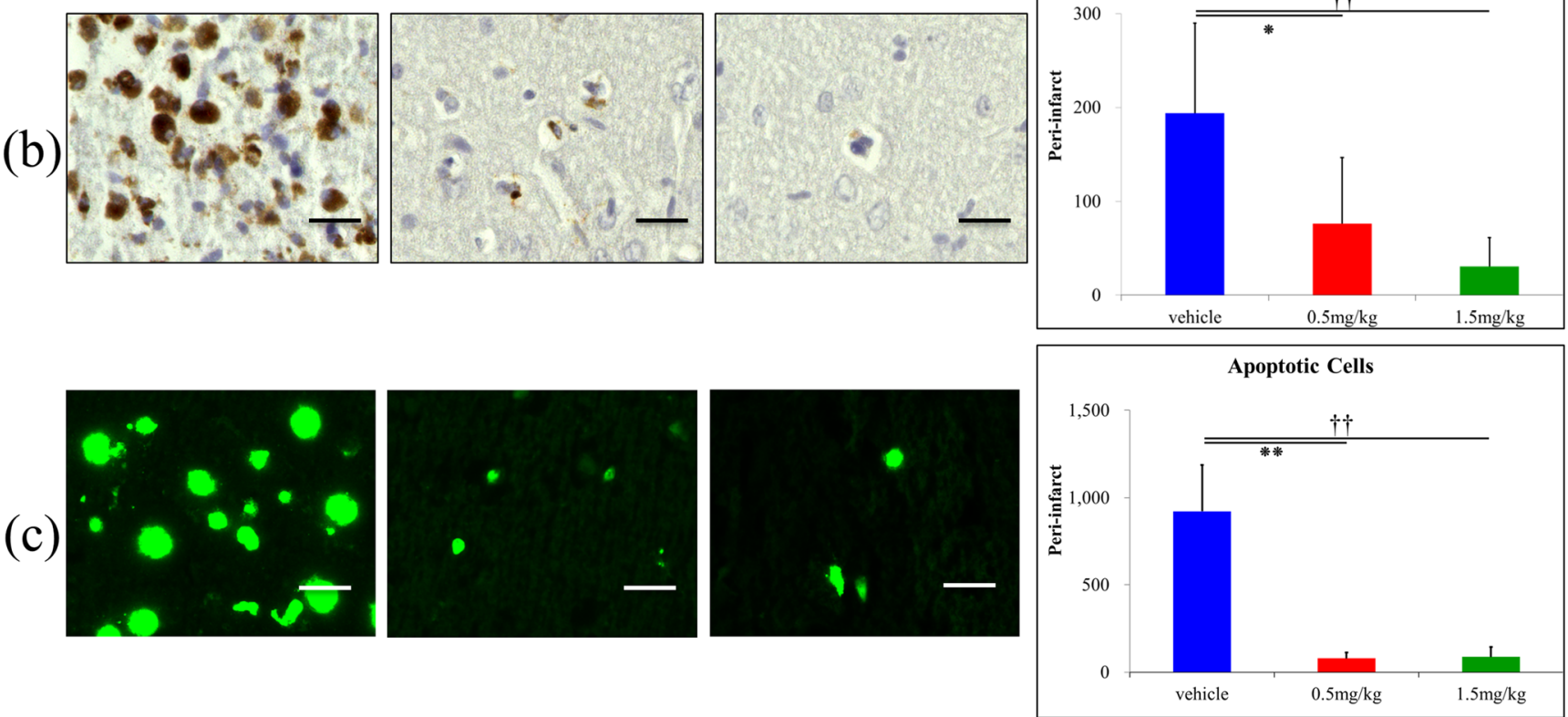

Fig. 2 Immunohistochemistry staining (day 7) and TUNEL staining (day 3 ) of transient middle cerebral artery occlusion rat model showing the extent of inflammation and cell death including apoptosis in the periinfarct areas. Iba-1-positive activated microglia (a) and macrophages (b) were dose-dependently decreased in the FTY720 groups ( $n=6$ per

group); $* P<0.05$ versus $0.5 \mathrm{mg} / \mathrm{kg}, \dagger \dagger P<0.01$ versus $1.5 \mathrm{mg} / \mathrm{kg}$. FTY720 significantly reduced the number of cell death including apoptotic cells in the FTY720-treated groups ( $n=6$ per group); $* * P<0.01$ versus $0.5 \mathrm{mg} / \mathrm{kg}, \dagger \dagger P<0.01$ versus $1.5 \mathrm{mg} / \mathrm{kg}$. Scale bar represents $25 \mu \mathrm{m}$. Higher magnification figure on the right upper corner $(\times 5)$

dependently decreased by both 3 and $10 \mathrm{kDa}$ dextran (Fig. $4 \mathrm{~b}, \mathrm{c})$. The effect of FTY720 and FTY720-P against BBB disruption was evaluated in an in vitro model. Leakage of Evans blue or FITC dextran was increased by the OGD condition (vehicle group). FTY720-P clearly ameliorated BBB permeability, although the difference was not significant. The effect of FTY720-P was completely abolished and even worsened by the existence of PTX (Fig. 5).

\section{FTY720/FTY720P Does Not Alter BBB mRNA Expression but Prevents Redistribution of BBB Components}

Quantitative real-time PCR analysis of tight and adherens junction markers was performed to elucidate the effect of FTY720 on the BBB. BBMVECs were treated with I/R injury for further analysis. The results revealed decreased mRNA levels of tight junction ( $\mathrm{ZO}-1$, occludin, claudin5) and adherens junction (VE-cadherin) markers. However, FTY720 and FTY720-P could not rescue the expression of these components as compared to levels in the vehicle group (Fig. 6). Therefore, BBB proteins and their distributions were further investigated (Fig. 7). Immunofluorescence analysis revealed ZO-1 and VE-cadherin, occludin, and claudin-5 proteins at the junctional cell surface (lamellipodia) of BBMVECs/rat brain microvascular endothelial cells cultured under normal conditions (Fig. 6; control), whereas I/R injury led to the redistribution and retraction of these proteins into the endothelial cytoplasm, which was thought to result in BBB disruption (Fig. 6; vehicle). Whereas vehicle and FTY720 did not ameliorate the redistribution of BBB components, FTY720-P significantly preserved ZO-1 and VE-cadherin proteins at the cell lamellipodia (Fig. 6a, b). The effect of FTY720-P on the protection of BBB components at the 
(a)

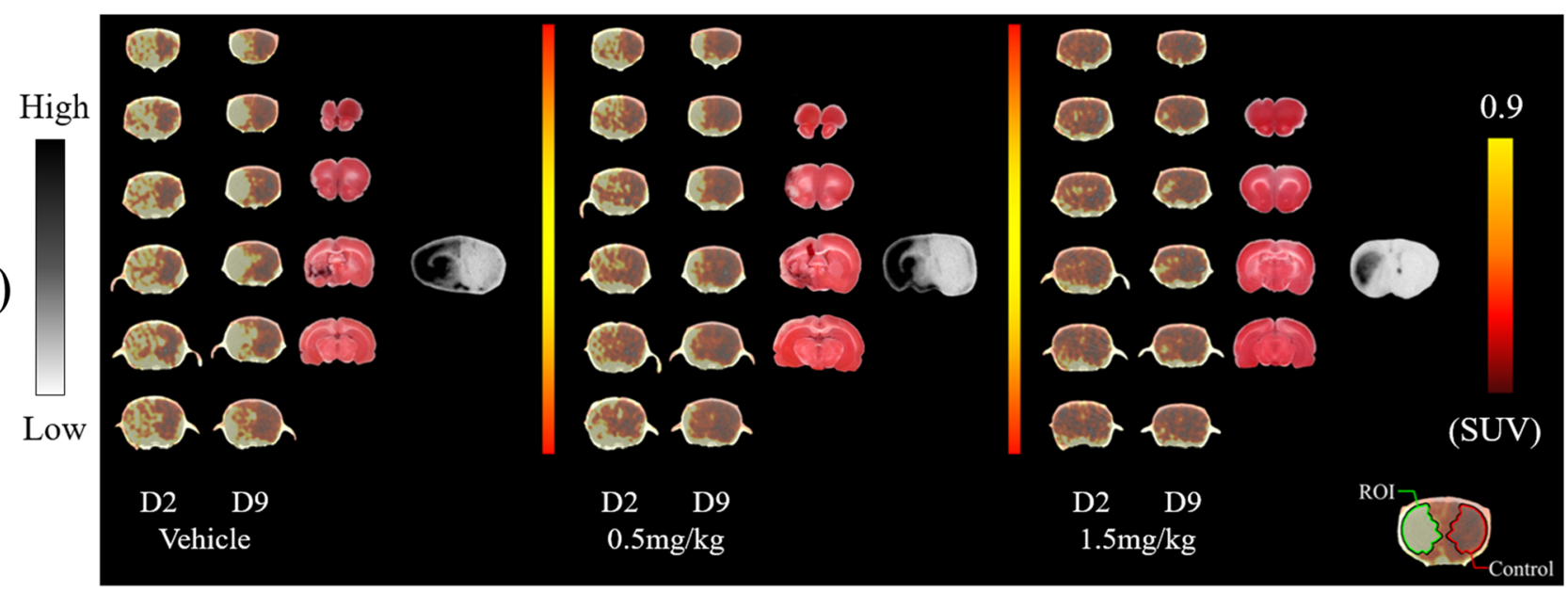

(b)
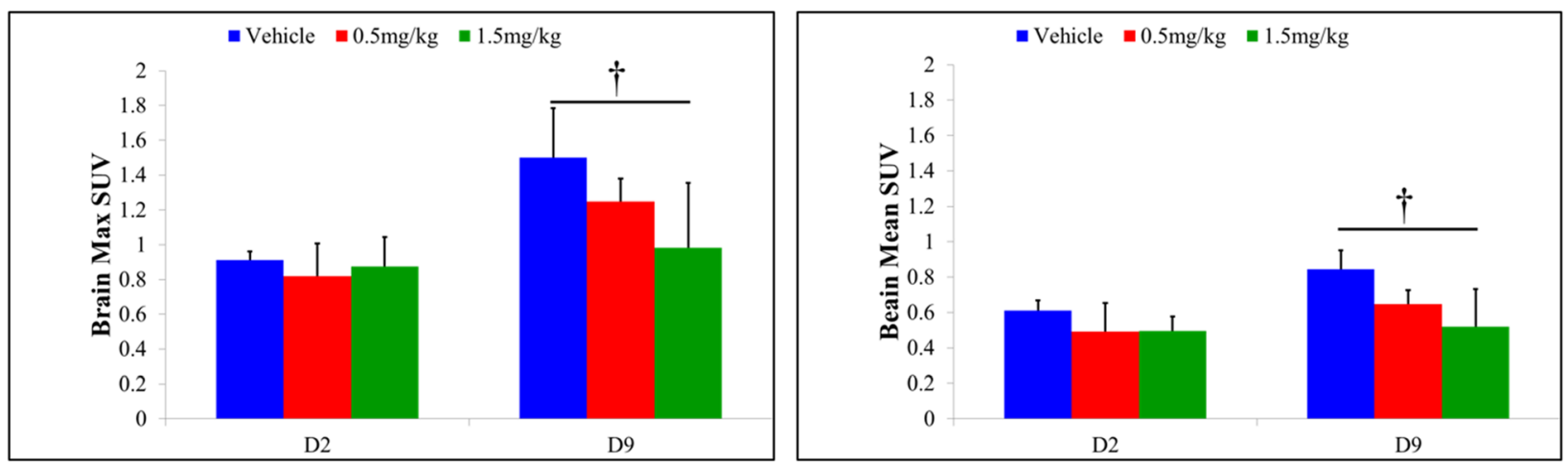

Fig. 3 Serial PET imaging of inflammation in transient middle cerebral artery occlusion rat model. a Left figures show representative PET images (yellow: ischemic area; and brown: healthy area) ( $n=4$ per group), 3,5triphenyltetrazolium chloride (TTC) staining (red represents healthy area and white represents ischemic area), and autoradiography (ARG; black represents ischemia and gray represents healthy area) of rat brains. Color bars indicate the scale of standardized uptake values (SUVs) in PET

lamellipodia was further confirmed by adding PTX, which antagonizes the major downstream pathway (Gi) of the S1P1 receptor; this completely abolished the effect of FTY720-P. In contrast, this preservation of junctional proteins was not observed for occludin and claudin-5 (Fig. 6c, d). We then determined whether the effect of FTY720-P occurred through $\mathrm{S}_{1} \mathrm{P}_{1}$ receptor binding. FTY720-P upregulated the expression of ERK1/2 signaling, which is downstream of the $\mathrm{S}_{1} \mathrm{P}_{1}$ receptor, as compared to that in the vehicle and FTY720 groups, whereas PTX again completely abolished downstream signaling from FTY720-P (Fig. 8).

\section{Discussion}

In the present study, we identified two novel protective mechanisms of FTY720 against I/R injury. First, FTY720 protected $\mathrm{BBB}$ integrity by preventing the redistribution of lamellipodia-located tight and adherens junctions, ZO-1 and images (right) and qualitative scale in ARG (left). b Graphs show the max SUV (left) and the mean SUV (right); $† P<0.05$ versus $1.5 \mathrm{mg} / \mathrm{kg}$. Vehicle-treated animals showed a significant increase in inflammation on day 9 compared to that on day 2, whereas FTY720 suppressed this effect. Particularly, in the high-dose group $(1.5 \mathrm{mg} / \mathrm{kg})$, nearly no exacerbation of inflammation was observed

VE-cadherin into the cytoplasm, which was mediated by $\mathrm{S}_{1} \mathrm{P}_{1}$ receptor signaling. Second, FTY720 alleviated neuroinflammation not in the acute phase but rather in the subacute phase, which was confirmed by serial PET imaging. In addition, FTY720 treatment led to a decrease in infarct size, improved neurological function, and ameliorated microglial/ macrophage activation and cell death.

During brain I/R injury, tight and adherens junction proteins are disrupted, which leads to a compromised BBB, allowing $\mathrm{T}$ and $\mathrm{B}$ cells to cross and subsequently causing inflammatory reactions [5, 21]. FTY720 has shown to stimulate the assembly of BBB junctional proteins such as VEcadherin and $\beta$-catenin under physiological conditions through Gi-coupled S1P receptors [32]. A previous report revealed that S1P administration induces the translocation of junction or adherens proteins into the lamellipodia and junctional regions, thereby enhancing barrier integrity [33]. These observations are in line with our findings showing that junctional proteins of ZO-1 and VE-cadherin are preserved from $\mathrm{I} / \mathrm{R}$ injury-induced translocation to the cytoplasm, resulting in 
(a)
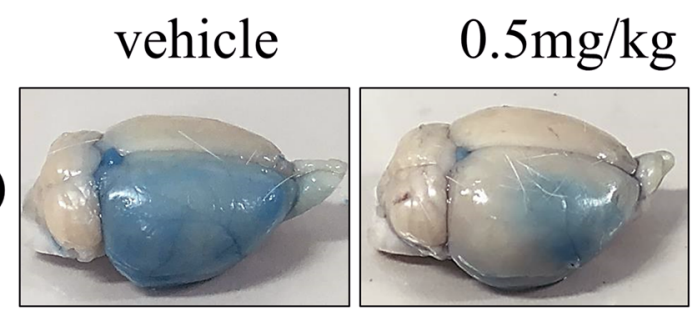

(b)
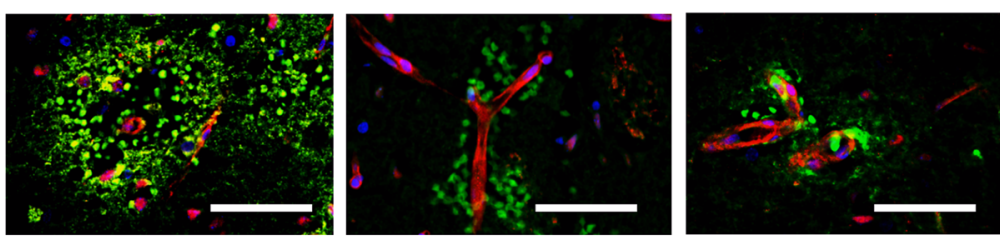

(c)
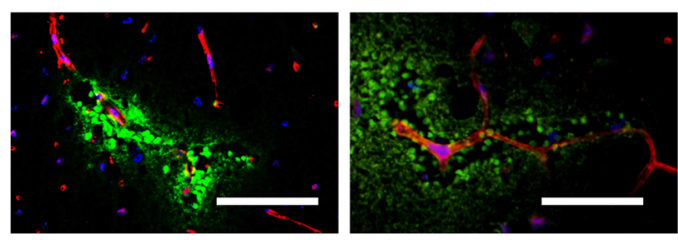
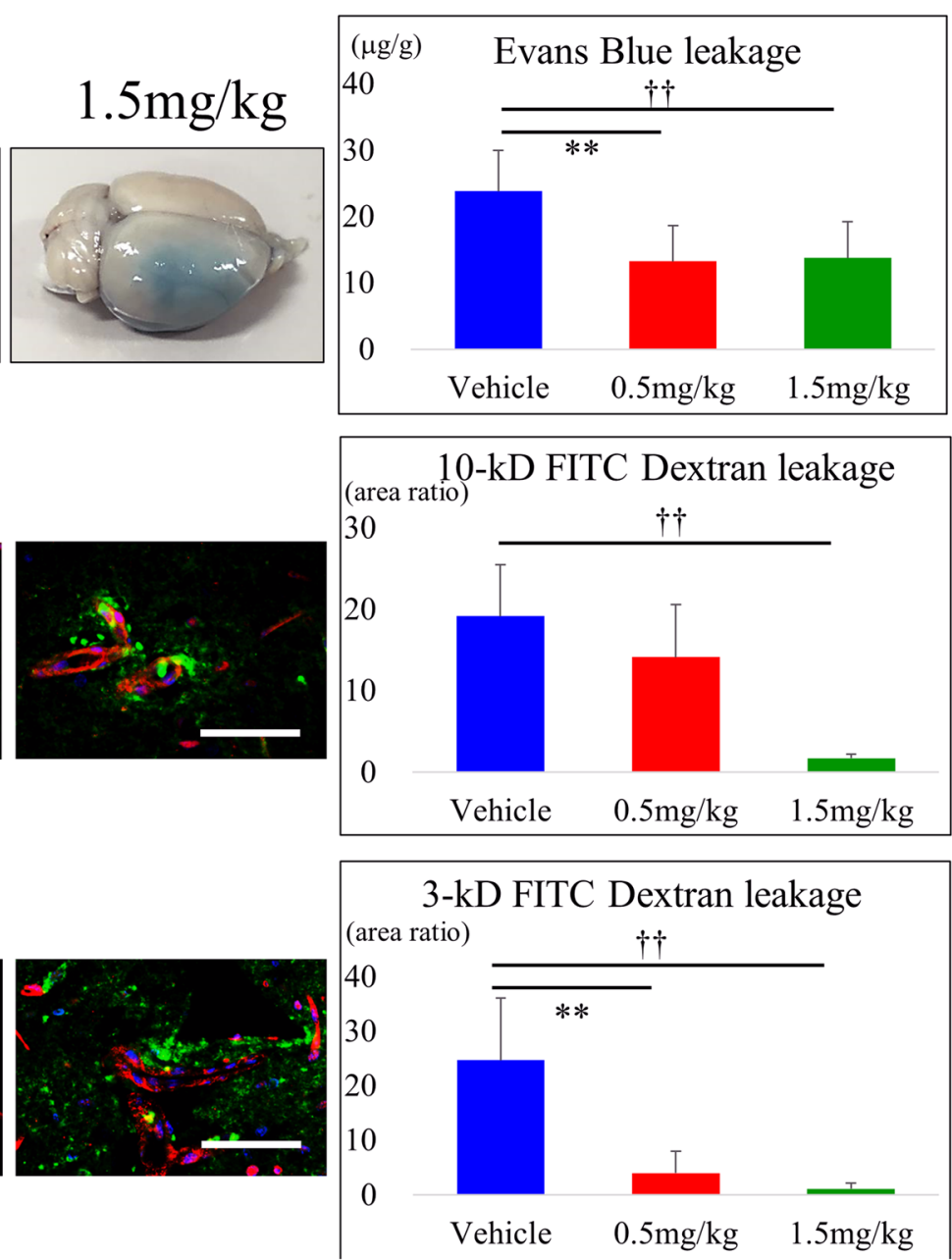

Fig. 4 Effect of FTY720 on blood-brain barrier (BBB) permeability in the brain of ischemia-reperfusion (I/R rats). After middle cerebral artery occlusion (MCAO)/reperfusion, Evans blue, 10-kD fluorescent dextran (FITC dextran), or 3-kD FITC dextran was infused by i.v. injection at day 1. The brains were isolated, and the supernatant was measured at $620 \mathrm{~nm}$ for absorbance using a spectrophotometer. Representative pictures and the spectrophotometry optical density of Evans blue was compared between groups (a) ( $n=6$ per group). Immunohistochemistry staining of brain microvessels (red) and leaked FITC (green) were compared (b; 10-kD FITC dextran, c; 3-kD FITC dextran). Scale bar represents $50 \mu \mathrm{m} . * * P<0.01$ versus $0.5 \mathrm{mg} / \mathrm{kg}, \dagger \dagger P<0.01$ versus $1.5 \mathrm{mg} / \mathrm{kg}$
BBB preservation. We found that FTY720 does not upregulate the mRNA expression of junctional proteins, suggesting that the de novo synthesis of junctional proteins was not the reason for the observed BBB preservation. Furthermore, the redistribution of these tight and adherens junctional proteins was abolished by PTX, which is the Gi receptor antagonist and main mediator of downstream signaling from the $\mathrm{S}_{1} \mathrm{P}_{1}$ receptor. This strongly suggests that this $\mathrm{BBB}$ protective mechanism is executed by $\mathrm{S}_{1} \mathrm{P}_{1}$ receptor signaling. However, unexpectedly, the protein distribution of occludin and claudin- 5 were not preserved by FTY720-P. These differences may have occurred because junctional proteins have different functional properties [34], but further studies are necessary to confirm this difference. Cannon et al. have previously reported that FTY720 acts on the brain endothelial cell S1P receptor 1 to reduce basal P-glycoprotein (P-gp), which functions as a ATP-driven drug efflux pump [35]. Our results and those of Cannon et al. suggest that TY720 prevents tight junction disruption at the expense of reducing downregulation of P-gp transport. Our data suggest a new mechanism by which FTY720 protects against ischemic insults, in which FTY720 preserves the BBB tight and adherens junction on the cell lamellipodia and prevents them from redistributing to the cytoplasm. In contrast, there have been conflicting reports, suggesting that co-administration of FTY720 and recombinant tissue plasminogen activator (rt-PA) can exacerbate BBB disruption [36], or that TEER of endothelial cells exposed to IFN $\gamma$ and TNF $\alpha$ were not changed by FTY720-P administration [37]. Reports from Hla et al. also showed that FTY720 facilitates selective BBB permeability to small molecules under physiological conditions. These conflicting data indicate the complicated mechanisms associated with $\mathrm{BBB}$ function and role of FTY720 [38]. S1P is biosynthesized from ceramide and sphingosine, and the expression levels of these 

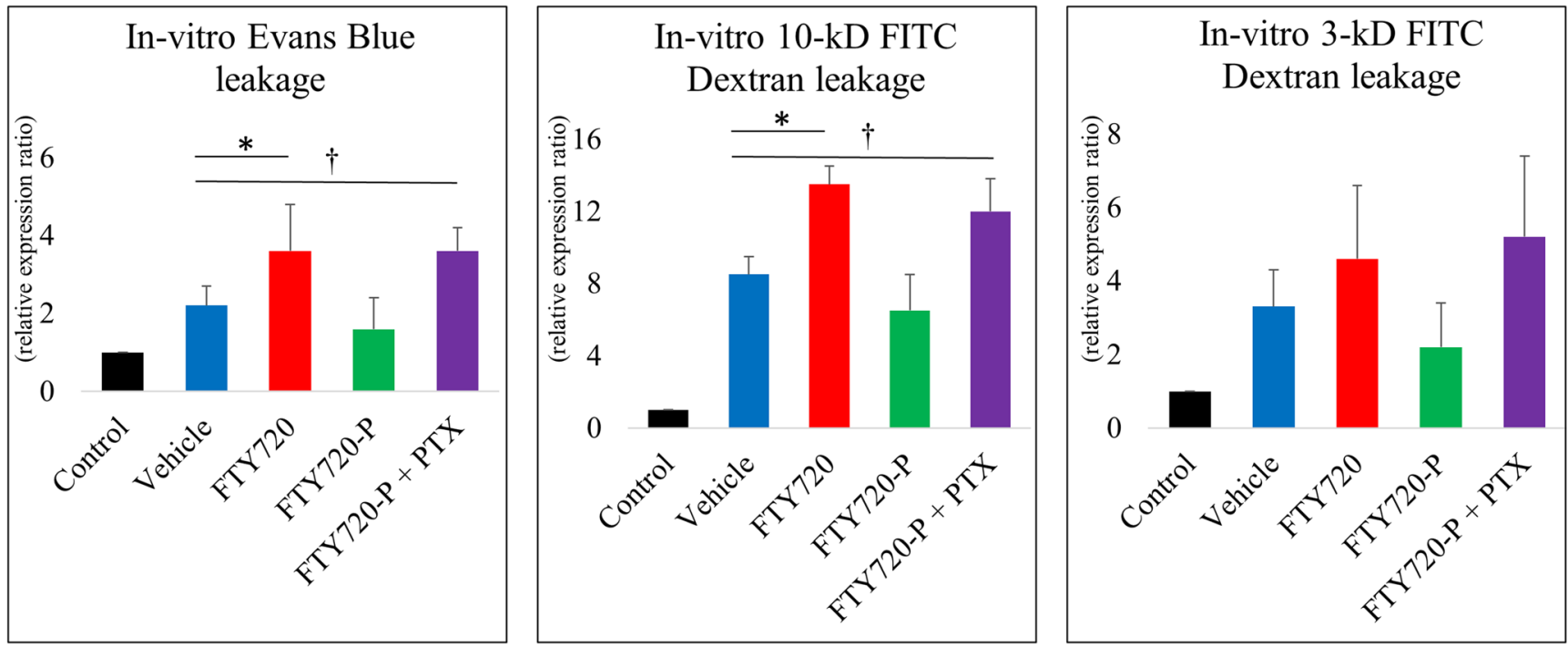

Fig. 5 Effect of FTY720/FTY720-P on BBB permeability in in vitro ischemia model. Relative expression levels were examined in a Evans blue, b 10-kD FITC dextran, and $\mathbf{c} 3$-kD FITC dextran. OGD condition (vehicle) disrupted BBB permeability compared to control. PTX appears to abolish the effect of FTY720-P. However, FTY720 or FTY720-P

(a)

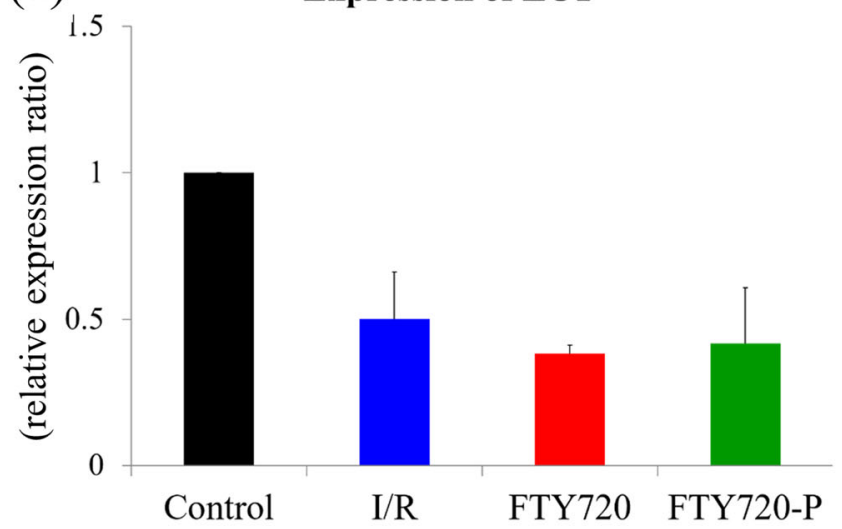

(c)

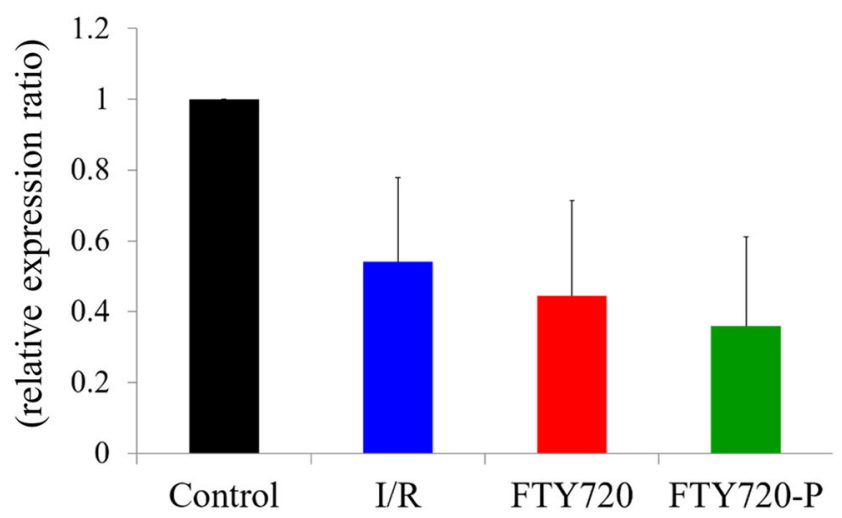

Fig. 6 mRNA expression of tight and adherens junctional proteins in transient middle cerebral artery occlusion rat model. mRNA expression for ZO-1 (a), Occludin (b), Claudin-5 (CLDN5) (c), and VE-cadherin (d) showed no protective role compared to OGD. FTY720 rather showed increased permeability in Evans blue and 10-kD FITC dextran compared to OGD. $* P<0.05$ vehicle versus FTY720, $\uparrow P<0.05$ vehicle versus FTY720-P + PTX (b)

Expression of Occludin

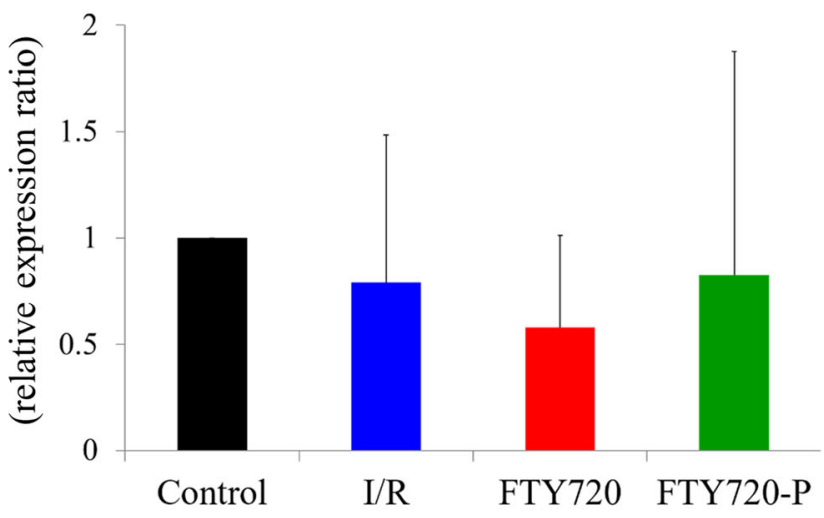

(d)

Expression of VE-Cadherin

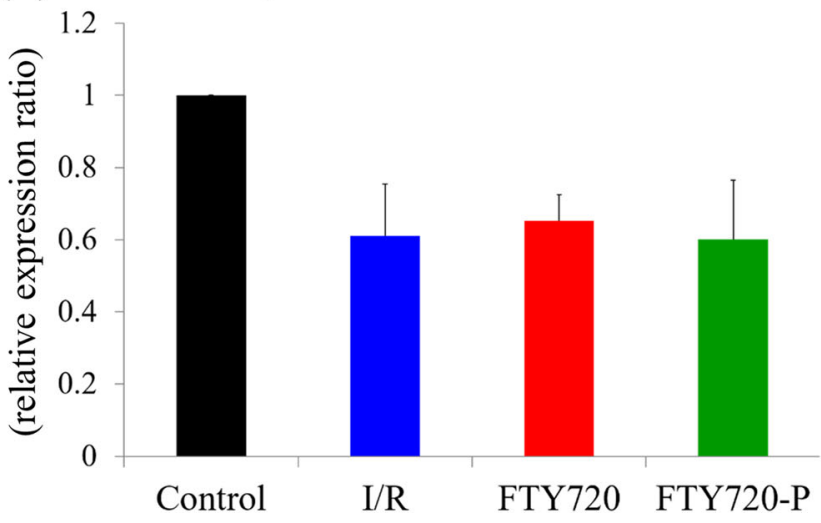

were evaluated. Ischemia-reperfusion (I/R) injury resulted in decreased mRNA expression; however, FTY720 and FTY720P did not restore the expression of mRNA 
(a) $\mathrm{ZO}-1$

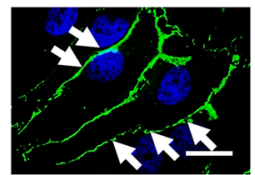

Control

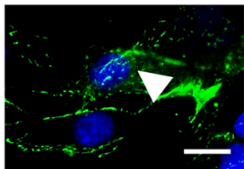

Vehicle

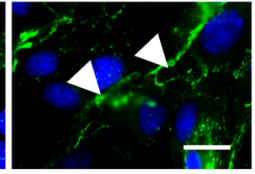

FTY720

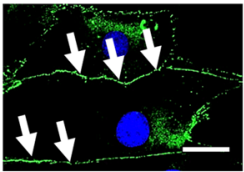

FTY720-P

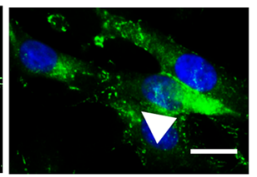

FTY720-P + PTX

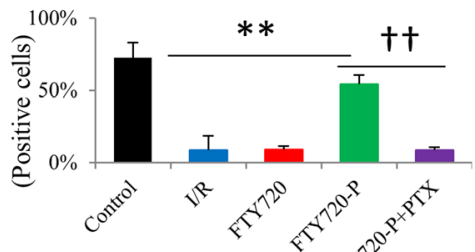

(b) VE-cadherin

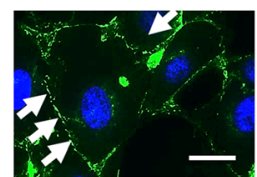

Control

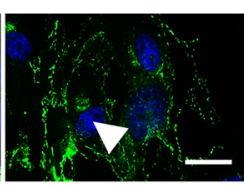

Vehicle

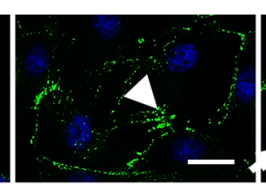

FTY720

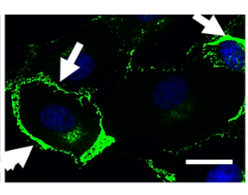

FTY720-P

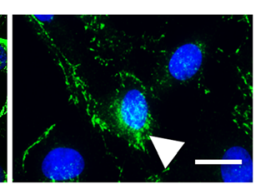

FTY720-P + PTX

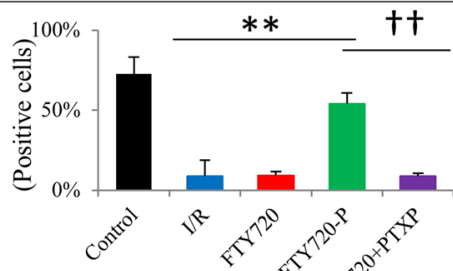

(c) Occludin

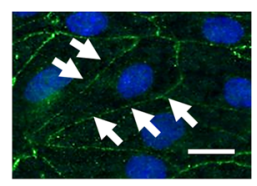

Control

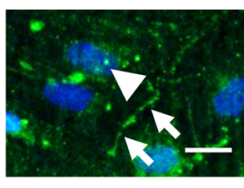

Vehicle

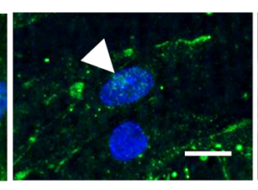

FTY720

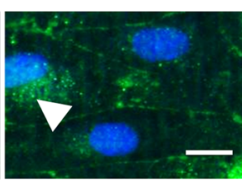

FTY720-P
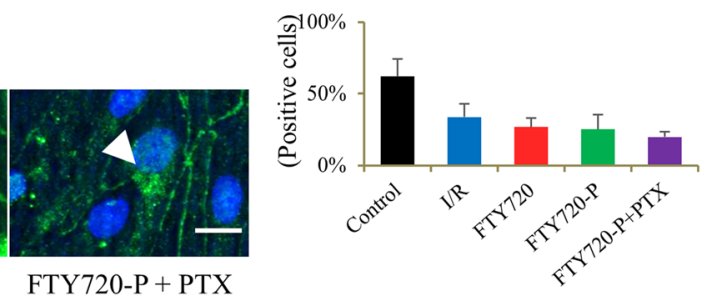

(d) Claudin-5

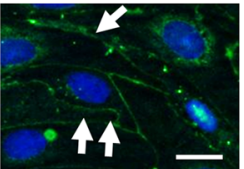

Control

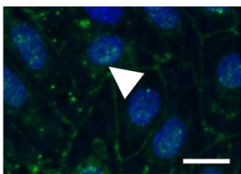

Vehicle

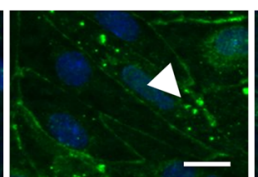

FTY720

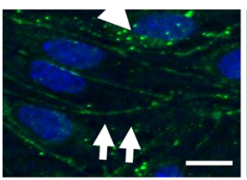

FTY720-P
FTY720-P + PTX

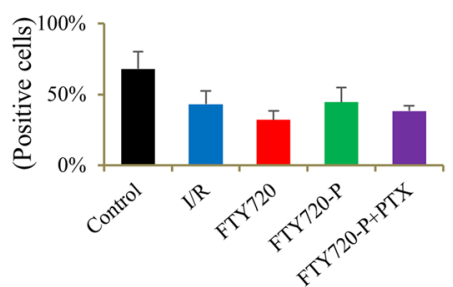

Fig. 7 Translocation of tight and adherens junction proteins in transient middle cerebral artery occlusion rat model (a ZO-1, b VE-cadherin, c occludin, d Claudin-5). Whereas ZO-1 and VE-cadherin were distributed at the lamellipodia under physiological conditions (a, b; control, arrow), disruption and retraction of these proteins into the cytoplasm was observed after ischemia-reperfusion (I/R) injury (a, b; vehicle, arrowhead). While FYT720 groups (a, b; FTY720, arrowhead) showed the same results as the vehicle group, specifically that junctional proteins were not preserved at the lamellipodia, FTY720-P successfully maintained the junctional proteins similar to that observed in the control group (a, b; FTY720-P; arrow). This effect was completely abolished by adding the $\mathrm{S}_{1} \mathrm{P}_{1}$ receptor antagonist, pertussis toxin (PTX) (a, b; FTY720-P+PTX, arrowhead). These protective roles of FTY720P was not observed in the occludin (c) and claudin-5 (d). Scale bar $=10 \mu \mathrm{m}$. $* P<0.05$ versus vehicle group, $* * P<0.01$ versus control group, $\uparrow \uparrow P<0.01$ versus PTX+ FTY720-P group

activated microglia in response to brain injury and inflammation and may be a good marker of neuroinflammation $[39,43]$. We found that vehicle-treated animals showed a significant increase in inflammation on day 9 compared to on day 2 , whereas FTY720 suppressed this effect. Particularly, in the high-dose group $(1.5 \mathrm{mg} / \mathrm{kg})$, nearly no exacerbation of inflammation was observed. This result may be used to optimize the timing to evaluate the inflammatory response with FTY720 in the future and may be a biomarker of successful treatment. The reason for the observed attenuation in inflammation at the later stage remains unclear, as inflammation is mediated by many critical factors including lymphocytes, leukocytes, microglia/macrophages, and the BBB. Lymphopenia has been reported as a primary mechanism for FTY720 both in vitro and in vivo and is considered one of the most normal physiological conditions but is highly expressed in 
Fig. 8 Western blot analysis was performed to elucidate the FTY720-P; ERK1/2 signaling was significantly increased in the FTY720-P group and completely abolished by PTX treatment $(n=$ 3 per group); $* P<0.05$ versus vehicle group, $* * P<0.01$ versus control group, $\dagger \dagger P<0.01$ versus PTX+FTY720-P group downstream signaling of
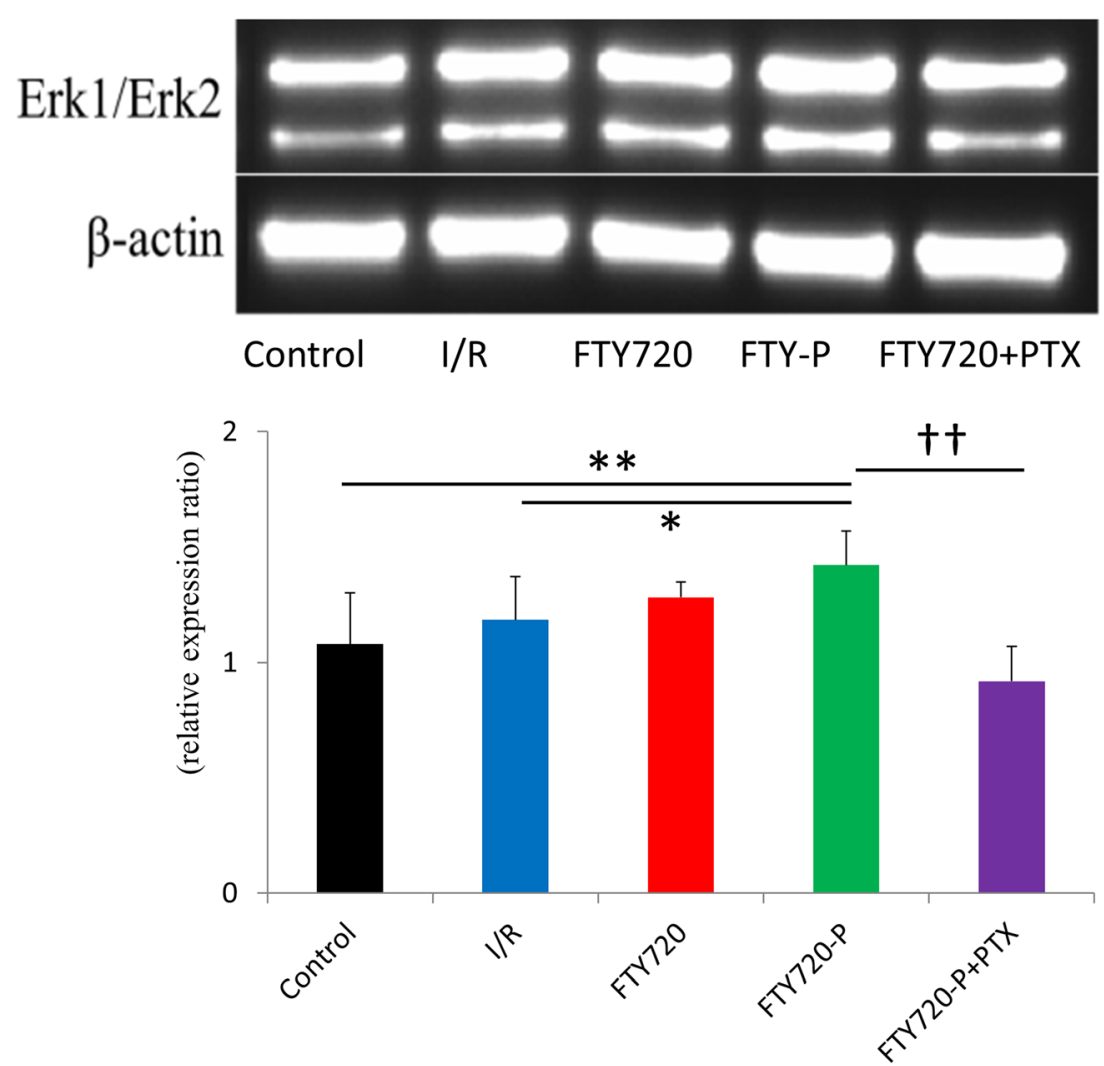

important therapeutic targets for this drug [15]. Administration of FTY720 in the early stage of ischemic stroke is clearly beneficial, as it induces lymphopenia, but this agent is considered to have a shorter half-life; thus, we suggest that this is not the only reason for this effect. We showed that FTY720 significantly reduces the number of activated microglia/macrophages and apoptotic cells. Interestingly, macrophages were reported to express the $\mathrm{S}_{1} \mathrm{P}_{2}$ receptor, whereas lymphocytes mainly harbor the $\mathrm{S}_{1} \mathrm{P}_{1}$ receptor [40]. FTY720 acts on four of five S1P receptors (S1P1, S1P3, S1P4, S1P5), demonstrating that it does not directly act on microglia. FTY720 was also reported to show no direct antiapoptotic effect on neural cells after ischemic insults [17]. Our result indicates that FTY720 significantly reduces microglia/ macrophage activation and cell death including apoptosis, not through the direct action of FTY720 on damaged cells, but rather through secondary damage in association with other factors including leukopenia and BBB preservation. This may also be confirmed by the fact that FTY720 could not improve outcomes following permanent $\mathrm{MCAO}$ but required reperfusion [44].

There were several limitations to this study. First, we were able to determine whether FTY720-P prevents the redistribution of tight and adherens junctional proteins, ZO-1 and VE-cadherin, to the lamellipodia, and FTY720 ameliorates BBB leakage in vivo. However, we were not able to directly demonstrate the relationship between the redistribution of $\mathrm{BBB}$ proteins and $\mathrm{BBB}$ leakage. Electron microscopy of the junctional regions or electrical cell substrate impedance assays, as well as measuring protease activities including MMP-9 may help to resolve this issue. Second, we could not directly demonstrate that preserving the BBB was associated with other results such as smaller infarct size, lower inflammation, and cell death. As lymphopenia is considered as one of the most prominent mechanisms underlying neurological preservation through FTY720, gene knockout mouse models or models in which leukocytes are abolished may be required to more precisely determine the effect of FTY720 on the BBB. Third, the drug was i.p.-injected from a technical perspective; however, when considering clinical use, i.v. injection and oral drug administration should also be applied. Fourth, FTY720 has increasingly been recognized to exert epigenetic effects independent of S1P receptor signaling [45-49]. We could not track the epigenetic effect of FTY720 in this study, particularly as it relates to ameliorating BBB functional deficiencies. Chromatin immunoprecipitation may be necessary to reveal this association.

In conclusion, the present study suggests that FTY720 can ameliorate $\mathrm{I} / \mathrm{R}$ injury, protecting the BBB by preventing the redistribution of junctional proteins into the cytoplasm and suppressing neuroinflammation at the subacute stage. Thus, FTY720 may be a promising candidate for alleviating I/R injury after revascularization. 
Funding Information This study was supported by Grant-in-Aids from the Ministry of Education, Science and Culture of Japan (16K19992), and AMED under Grant Number JP17bk0104045h.

\section{Compliance with Ethical Standards}

Conflict of Interest The authors declare that they have no conflicts of interest.

Research Involving Human Participants and/or Animals All institutional and national guidelines for the care and use of laboratory animals were followed.

Open Access This article is licensed under a Creative Commons Attribution 4.0 International License, which permits use, sharing, adaptation, distribution and reproduction in any medium or format, as long as you give appropriate credit to the original author(s) and the source, provide a link to the Creative Commons licence, and indicate if changes were made. The images or other third party material in this article are included in the article's Creative Commons licence, unless indicated otherwise in a credit line to the material. If material is not included in the article's Creative Commons licence and your intended use is not permitted by statutory regulation or exceeds the permitted use, you will need to obtain permission directly from the copyright holder. To view a copy of this licence, visit http://creativecommons.org/licenses/by/4.0/.

\section{References}

1. Feigin VL, Forouzanfar MH, Krishnamurthi R, Mensah GA, Connor M, Bennett DA, et al. Global and regional burden of stroke during 1990-2010: findings from the Global Burden of Disease Study 2010. Lancet. 2014;383(9913):245-54.

2. Saver JL, Goyal M, Bonafe A, Diener HC, Levy EI, Pereira VM, et al. Stent-retriever thrombectomy after intravenous t-PA vs. t-PA alone in stroke. N Engl J Med. 2015;372(24):2285-95. https://doi. org/10.1056/NEJMoa1415061.

3. Campbell BC, Mitchell PJ, Kleinig TJ, Dewey HM, Churilov L, Yassi N, et al. Endovascular therapy for ischemic stroke with perfusion-imaging selection. N Engl J Med. 2015;372(11):100918. https://doi.org/10.1056/NEJMoa1414792.

4. Goyal M, Demchuk AM, Menon BK, Eesa M, Rempel JL, Thornton J, et al. Randomized assessment of rapid endovascular treatment of ischemic stroke. N Engl J Med. 2015;372(11):101930. https://doi.org/10.1056/NEJMoa1414905.

5. Yenari MA, Han HS. Neuroprotective mechanisms of hypothermia in brain ischaemia. Nat Rev Neurosci. 2012;13(4):267-78. https:// doi.org/10.1038/nrn3174.

6. Sun MS, Jin H, Sun X, Huang S, Zhang FL, Guo ZN, et al. Free radical damage in ischemia-reperfusion injury: an obstacle in acute ischemic stroke after revascularization therapy. Oxidative Med Cell Longev. 2018;2018:3804979. https://doi.org/10.1155/2018/3804979.

7. Chiba K, Kataoka H, Seki N, Shimano K, Koyama M, Fukunari A, et al. Fingolimod (FTY720), sphingosine 1-phosphate receptor modulator, shows superior efficacy as compared with interferonbeta in mouse experimental autoimmune encephalomyelitis. Int Immunopharmacol. 2011;11(3):366-72. https://doi.org/10.1016/j. intimp.2010.10.005.

8. Mizuta K, Ohmori M, Miyashita F, Kitoh Y, Fujimura A, Mori M, et al. Effect of pretreatment with FTY720 and cyclosporin on ischaemia-reperfusion injury of the liver in rats. J Pharm Pharmacol. 1999;51(12):1423-8.
9. Man K, Ng KT, Lee TK, Lo CM, Sun CK, Li XL, et al. FTY720 attenuates hepatic ischemia-reperfusion injury in normal and cirrhotic livers. Am J Transplant. 2005;5(1):40-9. https://doi.org/10. 1111/j.1600-6143.2004.00642.x.

10. Delbridge MS, Shrestha BM, Raftery AT, El Nahas AM, Haylor J. FTY720 reduces extracellular matrix expansion associated with ischemia-reperfusion induced injury. Transplant Proc. 2007;39(10):2992-6. https://doi.org/10.1016/j.transproceed.2007. 04.027.

11. Kaudel CP, Frink M, Schmiddem U, Probst C, Bergmann S, Krettek C, et al. FTY720 for treatment of ischemia-reperfusion injury following complete renal ischemia; impact on long-term survival and T-lymphocyte tissue infiltration. Transplant Proc. 2007;39(2):499 502. https://doi.org/10.1016/j.transproceed.2006.12.010.

12. Wang M, Lu L, Liu Y, Gu G, Tao R. FTY720 attenuates hypoxiareoxygenation-induced apoptosis in cardiomyocytes. Exp Mol Pathol. 2014;97(2):218-24. https://doi.org/10.1016/j.yexmp.2014. 07.008 .

13. Goltz D, Huss S, Ramadori E, Buttner R, Diehl L, Meyer R. Immunomodulation by splenectomy or by FTY720 protects the heart against ischemia reperfusion injury. Clin Exp Pharmacol Physiol. 2015;42(11):1168-77. https://doi.org/10.1111/1440-1681. 12465.

14. van Vuuren D, Marais E, Genade S, Lochner A. The differential effects of FTY 720 on functional recovery and infarct size following myocardial ischaemia/reperfusion. Cardiovasc J Afr. 2016;27(6): 375-86. https://doi.org/10.5830/CVJA-2016-039.

15. Wei Y, Yemisci M, Kim HH, Yung LM, Shin HK, Hwang SK, et al. Fingolimod provides long-term protection in rodent models of cerebral ischemia. Ann Neurol. 2011;69(1):119-29. https://doi.org/ 10.1002/ana.22186

16. Nazari M, Keshavarz S, Rafati A, Namavar MR, Haghani M. Fingolimod (FTY720) improves hippocampal synaptic plasticity and memory deficit in rats following focal cerebral ischemia. Brain Res Bull. 2016;124:95-102. https://doi.org/10.1016/j. brainresbull.2016.04.004.

17. Kraft P, Gob E, Schuhmann MK, Gobel K, Deppermann C, Thielmann I, et al. FTY720 ameliorates acute ischemic stroke in mice by reducing thrombo-inflammation but not by direct neuroprotection. Stroke. 2013;44(11):3202-10. https://doi.org/10.1161/ STROKEAHA.113.002880.

18. Hasegawa Y, Suzuki H, Sozen T, Rolland W, Zhang JH. Activation of sphingosine 1-phosphate receptor-1 by FTY720 is neuroprotective after ischemic stroke in rats. Stroke. 2010;41(2):368-74. https://doi.org/10.1161/STROKEAHA.109.568899.

19. Brait VH, Tarrason G, Gavalda A, Godessart N, Planas AM. Selective sphingosine 1-phosphate receptor 1 agonist is protective against ischemia/reperfusion in mice. Stroke. 2016;47(12):3053-6. https://doi.org/10.1161/STROKEAHA.116.015371.

20. Gekka M, Abumiya T, Komatsu T, Funaki R, Kurisu K, Shimbo D, et al. Novel hemoglobin-based oxygen carrier bound with albumin shows neuroprotection with possible antioxidant effects. Stroke. 2018;49(8):1960-8. https://doi.org/10.1161/STROKEAHA.118. 021467.

21. Shimbo D, Abumiya T, Kurisu K, Osanai T, Shichinohe H, Nakayama N, et al. Superior microvascular perfusion of infused liposome-encapsulated hemoglobin prior to reductions in infarctions after transient focal cerebral ischemia. J Stroke Cerebrovasc Dis. 2017;26(12):2994-3003. https://doi.org/10.1016/j. jstrokecerebrovasdis.2017.07.026.

22. Shimbo D, Abumiya T, Shichinohe H, Nakayama N, Kazumata K, Houkin K. Post-ischemic intra-arterial infusion of liposomeencapsulated hemoglobin can reduce ischemia reperfusion injury. Brain Res. 2014;1554:59-66. https://doi.org/10.1016/j.brainres. 2014.01.038. 
23. Choe CU, Atzler D, Wild PS, Carter AM, Boger RH, Ojeda F, et al. Homoarginine levels are regulated by L-arginine:glycine amidinotransferase and affect stroke outcome: results from human and murine studies. Circulation. 2013;128(13):1451-61. https:// doi.org/10.1161/CIRCULATIONAHA.112.000580.

24. Chen J, Sanberg PR, Li Y, Wang L, Lu M, Willing AE, et al. Intravenous administration of human umbilical cord blood reduces behavioral deficits after stroke in rats. Stroke. 2001;32(11):2682-8.

25. Kaur H, Kumar A, Jaggi AS, Singh N. Pharmacologic investigations on the role of Sirt-1 in neuroprotective mechanism of postconditioning in mice. J Surg Res. 2015;197(1):191-200. https://doi.org/10.1016/j.jss.2015.03.010.

26. Mao Y, Kong Q, Li R, Zhang X, Gui Y, Li Y, et al. Heat shock protein $\mathrm{A} 12 \mathrm{~A}$ encodes a novel prosurvival pathway during ischaemic stroke. Biochim Biophys Acta. 2018;1864(5 Pt A):1862-72. https://doi.org/10.1016/j.bbadis.2018.03.006.

27. Lu C, Liu L, Chen Y, Ha T, Kelley J, Schweitzer J, et al. TLR2 ligand induces protection against cerebral ischemia/reperfusion injury via activation of phosphoinositide 3-kinase/Akt signaling. J Immunol. 2011;187(3):1458-66. https://doi.org/10.4049/ jimmunol.1003428.

28. Kawabori M, Kuroda S, Sugiyama T, Ito M, Shichinohe H, Houkin $\mathrm{K}$, et al. Intracerebral, but not intravenous, transplantation of bone marrow stromal cells enhances functional recovery in rat cerebral infarct: an optical imaging study. Neuropathology. 2012;32(3):21726. https://doi.org/10.1111/j.1440-1789.2011.01260.x.

29. Tan C, Zhao S, Higashikawa K, Wang Z, Kawabori M, Abumiya T, et al. [(18)F]DPA-714 PET imaging shows immunomodulatory effect of intravenous administration of bone marrow stromal cells after transient focal ischemia. EJNMMI Res. 2018;8(1):35. https:// doi.org/10.1186/s13550-018-0392-6.

30. Miyamoto M, Kuroda S, Zhao S, Magota K, Shichinohe H, Houkin $\mathrm{K}$, et al. Bone marrow stromal cell transplantation enhances recovery of local glucose metabolism after cerebral infarction in rats: a serial 18F-FDG PET study. J Nucl Med. 2013;54(1):145-50. https://doi.org/10.2967/jnumed.112.109017.

31. Magota K, Kubo N, Kuge Y, Nishijima K, Zhao S, Tamaki N. Performance characterization of the Inveon preclinical smallanimal PET/SPECT/CT system for multimodality imaging. Eur $\mathrm{J}$ Nucl Med Mol Imaging. 2011;38(4):742-52. https://doi.org/10. 1007/s00259-010-1683-y.

32. Sanchez T, Estrada-Hernandez T, Paik JH, Wu MT, Venkataraman $\mathrm{K}$, Brinkmann V, et al. Phosphorylation and action of the immunomodulator FTY720 inhibits vascular endothelial cell growth factorinduced vascular permeability. J Biol Chem. 2003;278(47):4728190. https://doi.org/10.1074/jbc.M306896200.

33. Lee JF, Zeng Q, Ozaki H, Wang L, Hand AR, Hla T, et al. Dual roles of tight junction-associated protein, zonula occludens- 1 , in sphingosine 1-phosphate-mediated endothelial chemotaxis and barrier integrity. J Biol Chem. 2006;281(39):29190-200. https://doi. org/10.1074/jbc.M604310200.

34. Umeda K, Matsui T, Nakayama M, Furuse K, Sasaki H, Furuse M, et al. Establishment and characterization of cultured epithelial cells lacking expression of ZO-1. J Biol Chem. 2004;279(43):44785-94. https://doi.org/10.1074/jbc.M406563200.

35. Cannon RE, Peart JC, Hawkins BT, Campos CR, Miller DS. Targeting blood-brain barrier sphingolipid signaling reduces basal P-glycoprotein activity and improves drug delivery to the brain. Proc Natl Acad Sci U S A. 2012;109(39):15930-5. https://doi. org/10.1073/pnas.1203534109.

36. Cai A, Schlunk F, Bohmann F, Kashefiolasl S, Brunkhorst R, Foerch C, et al. Coadministration of FTY720 and rt-PA in an experimental model of large hemispheric stroke-no influence on functional outcome and blood-brain barrier disruption. Exp Transl
Stroke Med. 2013;5(1):11. https://doi.org/10.1186/2040-7378-511.

37. Schuhmann MK, Bittner S, Meuth SG, Kleinschnitz C, Fluri F. Fingolimod (FTY720-P) does not stabilize the blood-brain barrier under inflammatory conditions in an in vitro model. Int J Mol Sci. 2015;16(12):29454-66. https://doi.org/10.3390/ijms161226177.

38. Yanagida K, Liu CH, Faraco G, Galvani S, Smith HK, Burg N, et al. Size-selective opening of the blood-brain barrier by targeting endothelial sphingosine 1-phosphate receptor 1. Proc Natl Acad Sci U S A. 2017;114(17):4531-6. https://doi.org/10.1073/pnas. 1618659114.

39. Chen MK, Guilarte TR. Translocator protein $18 \mathrm{kDa}$ (TSPO): molecular sensor of brain injury and repair. Pharmacol Ther. 2008;118(1):1-17. https://doi.org/10.1016/j.pharmthera.2007.12. 004.

40. Michaud J, Im DS, Hla T. Inhibitory role of sphingosine 1phosphate receptor 2 in macrophage recruitment during inflammation. J Immunol. 2010;184(3):1475-83. https://doi.org/10.4049/ jimmunol.0901586.

41. Ory D, Planas A, Dresselaers T, Gsell W, Postnov A, Celen S, et al. PET imaging of TSPO in a rat model of local neuroinflammation induced by intracerebral injection of lipopolysaccharide. Nucl Med Biol. 2015;42(10):753-61. https://doi.org/10.1016/j.nucmedbio. 2015.06.010.

42. Papadopoulos V, Baraldi M, Guilarte TR, Knudsen TB, Lacapere JJ, Lindemann P, et al. Translocator protein (18kDa): new nomenclature for the peripheral-type benzodiazepine receptor based on its structure and molecular function. Trends Pharmacol Sci. 2006;27(8):402-9. https://doi.org/10.1016/j.tips.2006.06.005.

43. Jacobs AH, Tavitian B, Consortium IN. Noninvasive molecular imaging of neuroinflammation. J Cereb Blood Flow Metab. 2012;32(7):1393-415. https://doi.org/10.1038/jcbfm.2012.53.

44. Liesz A, Sun L, Zhou W, Schwarting S, Mracsko E, Zorn M, et al. FTY720 reduces post-ischemic brain lymphocyte influx but does not improve outcome in permanent murine cerebral ischemia. PLoS One. 2011;6(6):e21312. https://doi.org/10.1371/journal.pone. 0021312.

45. Baer A, Colon-Moran W, Bhattarai N. Characterization of the effects of immunomodulatory drug fingolimod (FTY720) on human $\mathrm{T}$ cell receptor signaling pathways. Sci Rep. 2018;8(1):10910. https://doi.org/10.1038/s41598-018-29355-0.

46. Gardner NM, Riley RT, Showker JL, Voss KA, Sachs AJ, Maddox JR, et al. Elevated nuclear and cytoplasmic FTY720-phosphate in mouse embryonic fibroblasts suggests the potential for multiple mechanisms in FTY720-induced neural tube defects. Toxicol Sci. 2016;150(1):161-8. https://doi.org/10.1093/toxsci/kfv321.

47. Hait NC, Wise LE, Allegood JC, O'Brien M, Avni D, Reeves TM, et al. Active, phosphorylated fingolimod inhibits histone deacetylases and facilitates fear extinction memory. Nat Neurosci. 2014;17(7):971-80. https://doi.org/10.1038/nn.3728.

48. Leo A, Citraro R, Amodio N, De Sarro C, Gallo Cantafio ME, Constanti A, et al. Fingolimod exerts only temporary antiepileptogenic effects but longer-lasting positive effects on behavior in the WAG/Rij rat absence epilepsy model. Neurotherapeutics. 2017;14(4):1134-47. https://doi.org/10.1007/ s13311-017-0550-y.

49. Segura-Ulate I, Yang B, Vargas-Medrano J, Perez RG. FTY720 (Fingolimod) reverses alpha-synuclein-induced downregulation of brain-derived neurotrophic factor mRNA in OLN-93 oligodendroglial cells. Neuropharmacology. 2017;117:149-57. https://doi.org/ 10.1016/j.neuropharm.2017.01.028.

Publisher's Note Springer Nature remains neutral with regard to jurisdictional claims in published maps and institutional affiliations. 